\title{
Uma Abordagem Baseada em Ontologias para Modelagem Semântica e Geográfica de Sites de Conteúdo Social no Contexto dos Setores Públicos
}

\author{
Luciana Cavalcante de Menezes, Hugo Feitosa de Figueirêdo, Ricardo Madeira \\ Fernandes, Tiago Eduardo da Silva, Cláudio de Souza Baptista
}

Departamento de Sistemas e Computação - Universidade Federal de Campina Grande (UFCG) - Rua Aprígio Veloso SN - Campina Grande - PB - Brasil

\{luciana, hugoff, madeira, tsilva, baptista\} edsc.ufcg.edu.br

\begin{abstract}
In the Web 2.0 users may freely contribute with information that concern about a variety of topics. Hence, public agencies can take advantage of this fact by creating communication channels with society. However, it is necessary to provide a properly environment to insert these agencies into social content sites. This paper aims to present an ontology-based approach for semantic and geographical modeling of social content sites in the context of public agencies, and a prototype built using the proposed model.
\end{abstract}

Resumo. A Web 2.0 permite que os usuários publiquem livremente informações sobre diversos tópicos. Os setores públicos podem tirar vantagem deste fato criando canais de comunicação com a sociedade. Entretanto é necessário prover um ambiente adequado para inserir estes setores em sites de conteúdo social. Esse artigo apresenta uma abordagem baseada em ontologias para a modelagem semântica e geográfica de sites de conteúdo social para setores públicos e um protótipo construído utilizando o modelo proposto.

\section{Introdução}

O surgimento da Web Social, mais conhecida como Web 2.0, modificou bastante a forma como as pessoas se relacionam e compartilham informações. As ferramentas criadas com a tecnologia da Web social, tais como blogs, wikis e as redes sociais facilitam a produção, o consumo e a propagação dessas informações [Sheth and Nagarajan 2009]. Neste contexto, os sites de conteúdo social se destacam por integrarem em um mesmo ambiente, informações tradicionais com funcionalidades presentes nas redes sociais [Amer-Yahia et al. 2009A], tornando a experiência dos usuários na Web ainda mais enriquecedora, uma vez que eles podem contribuir com opiniões e informações para o site e também compartilhá-las com outras pessoas interessadas no mesmo assunto ou pertencentes a sua rede de amizade.

Devido à participação das pessoas, os sites de conteúdo social se tornaram um ambiente propício para a disseminação de opiniões críticas a respeito de acontecimentos do dia-a-dia. Sendo assim, pode-se observar nestes sites um potencial para o incentivo da comunicação entre a sociedade e os setores privados e, principalmente, públicos.

No Brasil, alguns setores públicos utilizam o mecanismo do orçamento participativo, que são reuniões realizadas com alguns líderes de comunidades, para se 
comunicar com a sociedade. Nestas reuniões são ouvidas as necessidades da comunidade com o objetivo de priorizar o destino dos recursos financeiros disponíveis para as áreas mais necessitadas. Neste caso, a utilização de um site de conteúdo social, onde pessoas pudessem opinar sobre os principais problemas enfrentados pela sua comunidade, ampliaria ainda mais a interação entre o setor público e a sociedade. Outros setores públicos, que ainda não possuem mecanismos de interação popular, como é o Tribunal de Contas dos Estados, poderiam por exemplo, criar um site para que a sociedade fiscalizasse a veracidade das obras que estariam em andamento nos Estados, enviando fotos ou vídeos sobre construções que existem apenas no papel.

Além da informação tradicional, Goodchild [Goodchild 2007] salienta a importância da aplicação da informação nos serviços da Web 2.0. No modelo proposto por ele, os participantes são denominados 'cidadãos como sensores', uma vez que eles são responsáveis por fornecer detalhes a respeito do local de suas observações e experiências voluntariamente. Atualmente este modelo tornou-se uma tendência, principalmente após o surgimento do conceito de mashups de mapas, que são aplicações que combinam informações e funcionalidades de diferentes fontes online por meio de interfaces acessíveis ao público [Merrill 2006]. Portanto, a junção da informação tradicional com a geográfica ajuda a melhorar a percepção do cidadão a respeito do seu ambiente social, uma vez que possibilita a realização de buscas espaciais na informação.

Neste contexto, propomos uma abordagem baseada em ontologias para realizar a modelagem das interações entre o cidadão e o setor público em sites de conteúdo social, considerando aspectos semânticos e geográficos, com o objetivo de possibilitar que máquinas sejam capazes de interpretar melhor as informações presentes no site, propiciando ao usuário uma experiência mais enriquecedora, uma vez que ele terá resultados mais precisos em suas buscas por “o quê?" e "onde?".

Para verificar na prática a utilização da ontologia [Guarino 1996] proposta desenvolvemos um protótipo, denominado SocialSupervisor, cujo objetivo é estreitar a comunicação entre órgãos públicos, responsáveis pela supervisão de obras, e a sociedade, possibilitando 1) ao setor público expor de maneira transparente a informação a respeito do progresso das obras de determinada região geográfica; 2) ao cidadão atuar como um supervisor das obras públicas, através do envio de informações em formato multimídia; 3 ) a sociedade ter acesso fácil a informação a respeito de uma determinada obra pública.

$\mathrm{O}$ restante deste artigo é organizado da seguinte forma. Na seção 2 são apresentados os trabalhos relacionados. A seção 3 mostra a modelagem semântica e geográfica da abordagem proposta. O protótipo de implementação do modelo é apresentado na seção 4 e na seção 5 são discutidos aspectos relevantes da implementação do mesmo. Na seção 6 são apresentadas motivações para novas aplicações. Finalmente, na seção 7 é apresentada uma conclusão a respeito do que foi abordado neste artigo e aponta novos trabalhos a realizar.

\section{Trabalhos Relacionados}

Existem diversos trabalhos que mostram ferramentas de software cujo objetivo é melhorar a comunicação entre o cidadão e o governo. Recentemente, Teerling e Pieterson [Teerling and Pieterson 2009] apresentaram um estudo que mostra como 
conduzir as pessoas na utilização deste tipo de canal de comunicação, uma vez ele não é explorado de forma eficiente pela maioria das delas.

O projeto Smartocracy [Rodriguez et al. 2007] tem como objetivo utilizar um modelo problema-solução no qual os usuários podem enviar seus problemas e soluções para a comunidade. As propostas enviadas recebem votos para indicar qual solução, a comunidade considera ser a melhor, para um determinado problema. Esse trabalho também apresenta uma rede social baseada em confiança para representar as relações entre os usuários. Entretanto, esse processo de confiança pode ser melhorado através da utilização da informação geográfica do problema proposto pela comunidade e da localização dos usuários que votam nas possíveis soluções disponíveis.

Boldi et al. [Boldi et al. 2009] também trata a questão da confiança através da utilização de votos em redes sociais mediadas de forma eletrônica. Eles propõem que o sistema de votação nestas redes seja configurado de forma a permitir que o usuário expresse sua preferência a apenas uma pessoa com o qual ele tenha um relacionamento direto.

O Wiki Government [Noveck 2009] é um projeto que utiliza a tecnologia dos Wikis para criar um governo colaborativo e democrático. Embora este projeto permita que decisões públicas sejam realizadas através da participação pública, ele centra-se apenas no campo específico do processo de exame de patentes.

Maciel et al. [Maciel et al. 2009] apresenta um estudo de caso piloto para o projeto denominado 'Democratic Citizenship Community', que visa fornecer recursos de interação e comunicação, tais como perfis dos cidadãos, debate, voto, informação de bibliotecas, socialização do espaço e ajuda dos usuários. Novamente, este projeto não considera a informação a respeito da localização geográficas dos usuários e dos problemas.

Hiendiger et al. [Heidinger et al 2009] propõe uma ferramenta Web para promover a avaliação de impacto de colaboração, visando melhor avaliar as políticas, normas ou regulações que estão sendo desenvolvidas pelos legisladores. Nos experimentos realizados, foi descoberto que o sucesso da ferramenta dependia da interface e da usabilidade. Certamente, a utilização de capacidades espaciais na interface irá melhorar ainda mais os resultados.

No campo da descoberta e gerenciamento da informação, o trabalho de Amer Yahia et al. [Amer-Yahia et al. 2009B] apresenta uma arquitetura lógica para tratar dos desafios de se gerenciar e descobrir a informação presente em sites de conteúdo social. A arquitetura proposta, intitulada de Social Scope, consiste em três camadas: gerenciamento de conteúdo, descoberta da informação e apresentação da informação. Entretanto, eles não abordam a questão da dimensão espacial.

Naaman [Naaman 2009] é um dos primeiros trabalhos a explorar questões espaço temporais em redes sociais. Monclar et al [Monclar et al. 2009] propôs uma ferramenta denominada MEK (Mobile Exchange of Knowledge) que tem o objetivo de prover uma infraestrutura móvel para a troca de conhecimento entre pessoas que compartilham do mesmo interesse, levando em consideração os aspectos geográficos das pessoas envolvidas. Todavia, esta abordagem não foi aplicada ao contexto da comunicação entre os cidadãos e o governo. 
Em nenhum dos trabalhos acima citados é apresentado um modelo de ontologias para mapear as interações dos usuários que contribuem com a Web Social e os setores públicos.

\section{Modelagem Semântica e Geográfica do Site de Conteúdo Social}

A modelagem semântica e geográfica do site de conteúdo social baseou-se em diversos modelos já desenvolvidos, e amplamente utilizados no âmbito da web semântica [Berners-Lee et al 2001]. Estes modelos foram agrupados, e quando necessário, extendido para possibilitar o mapeamento das interações entre o cidadão e o setor público.

Na representação da semântica utilizou-se a ontologia MOAT - Meaning of a Tag, proposta por Passant e Laublet [Passant and Laublet 2008], que é uma subclasse da Tag Ontology de Newman [Newman 2005] e que também se baseia nos vocabulários SIOC [SIOC] e FOAF [FOAF]. Ela prove uma classe denominada Meaning que possibilita associar semântica as tags.

Para realizar a representação geográfica no site de conteúdo social foi utilizada a GeoOWL [GeoOWL], uma ontologia desenvolvida pelo grupo W3C que usa o vocabulário GeoRSS [GeoRSS] na modelagem das classes e propriedades geográficas. Esta ontologia suporta o conceito de pontos (que contém um par de coordenadas), linhas (possuindo dois ou mais pares de coordenadas), boxes (que possuem exatamente dois pares de coordenadas) e polígonos (representados com no mínimo quatro pares de coordenadas).

As ontologias MOAT e GeoOWL foram combinadas para gerar a modelagem semântica e geográfica. Além destas ontologias, foram utilizados: o vocabulário FOAF, para representar os usuários do sistema e suas redes sociais e o vocabulário SIOC para integrar as informações publicadas ao site de conteúdo social. Na próxima subseção apresentaremos a ScsOWL, a ontologia criada para realizar a modelagem semântica e geográfica de sites de conteúdo social.

\subsection{Ontologia ScsOWL}

$\mathrm{Na}$ ontologia ScsOWL a modelagem semântica e geográfica do site de conteúdo social é feita utilizando a classe tags:RestrictedTagging pertencente a Tag Ontology, uma vez que com ela, é possível mapear o relacionamento entre um recurso, um usuário e uma única tag. A propriedade moat:tagMeaning realiza a ligação entre a tag criada e uma URI que representa seu significado. Para relacionar a tag com sua localização geográfica criamos a propriedade scs:has_location que liga a tag a uma a uma instancia da classe gml:_Geometry, presente na GeoOWL.

Para representação do site foi criada a classe scs:SocialContentSite que é uma subclasse da classe sioc:Site e que possui a propriedade scs:has_location para indicar seu escopo geográfico. Ao scs:SocialContentSite também podem ser atribuídas tags:RestrictedTagging.

Os usuários modelados são de três tipos: scs:Anonymous, scs:OwnerUser e scs:CitizenUser. Os dois últimos são subclasses da classe foaf:Person e se relacionam com o sioc:User através da propriedade foaf:holdsAccount e apenas eles podem criar 
tags. Todos os usuários podem publicar posts, entretanto apenas os usuários scs:OwnerUser e scs:CitizenUser podem colocar no site outros tipos de recursos.

Para mapear posts que representam reclamações dos usuários, foi criado o scs:Complaint, que é uma subclasse de sioc:Post e nele é possível associar um escopo geográfico através da propriedade scs:has_location. scsOWL.

$\mathrm{Na}$ Figura 1 é apresentada uma representação simplificada da ontologia

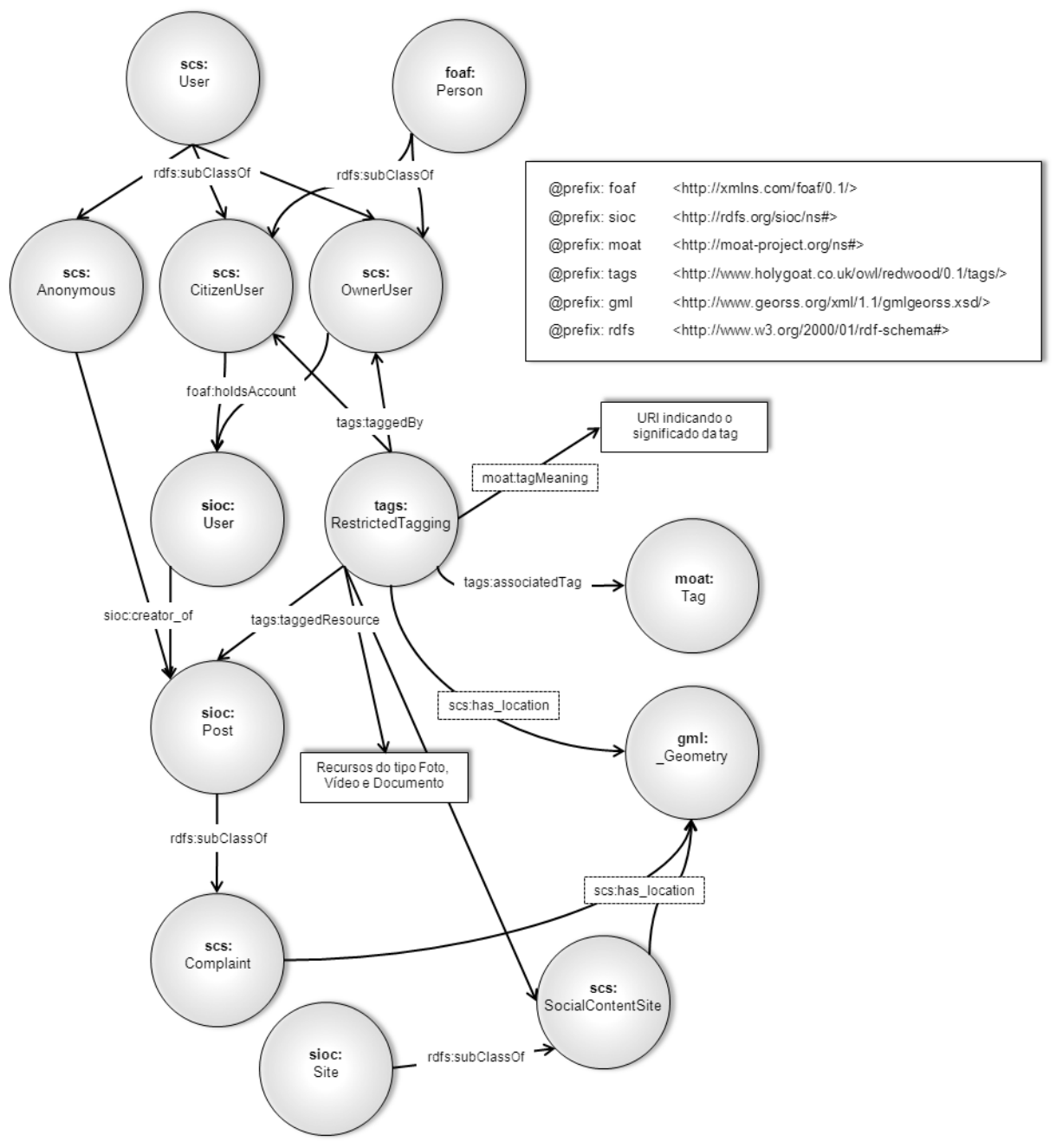

Figura 1: Representação simplificada da ontologia scsOWL

\section{SocialSupervisor: Implementando o modelo proposto}

O SocialSupervisor é um site de conteúdo social, no qual usuários autorizados podem cadastrar obras públicas e anexar a elas arquivos multimídia que identificam seu progresso. Além disso, a sociedade pode acessar o site através da Internet e realizar denúncias, comentários ou simplesmente ter uma visão geral das obras públicas que estão sendo construídas ou já foram concluídas em determinada região geográfica. Em sua implementação a ontologia scsOWL foi utilizada para realizar toda a modelagem semântica e geográfica do site. 
Para definir a arquitetura do SocialSupervisor foi utilizado o modelo de três camadas, com o objetivo de permitir maior flexibilidade ao sistema, já que neste modelo a excelente separação entre as camadas possibilita que cada uma seja alterada independente da outra. Na Figura 2 são mostradas as três camadas do sistema.

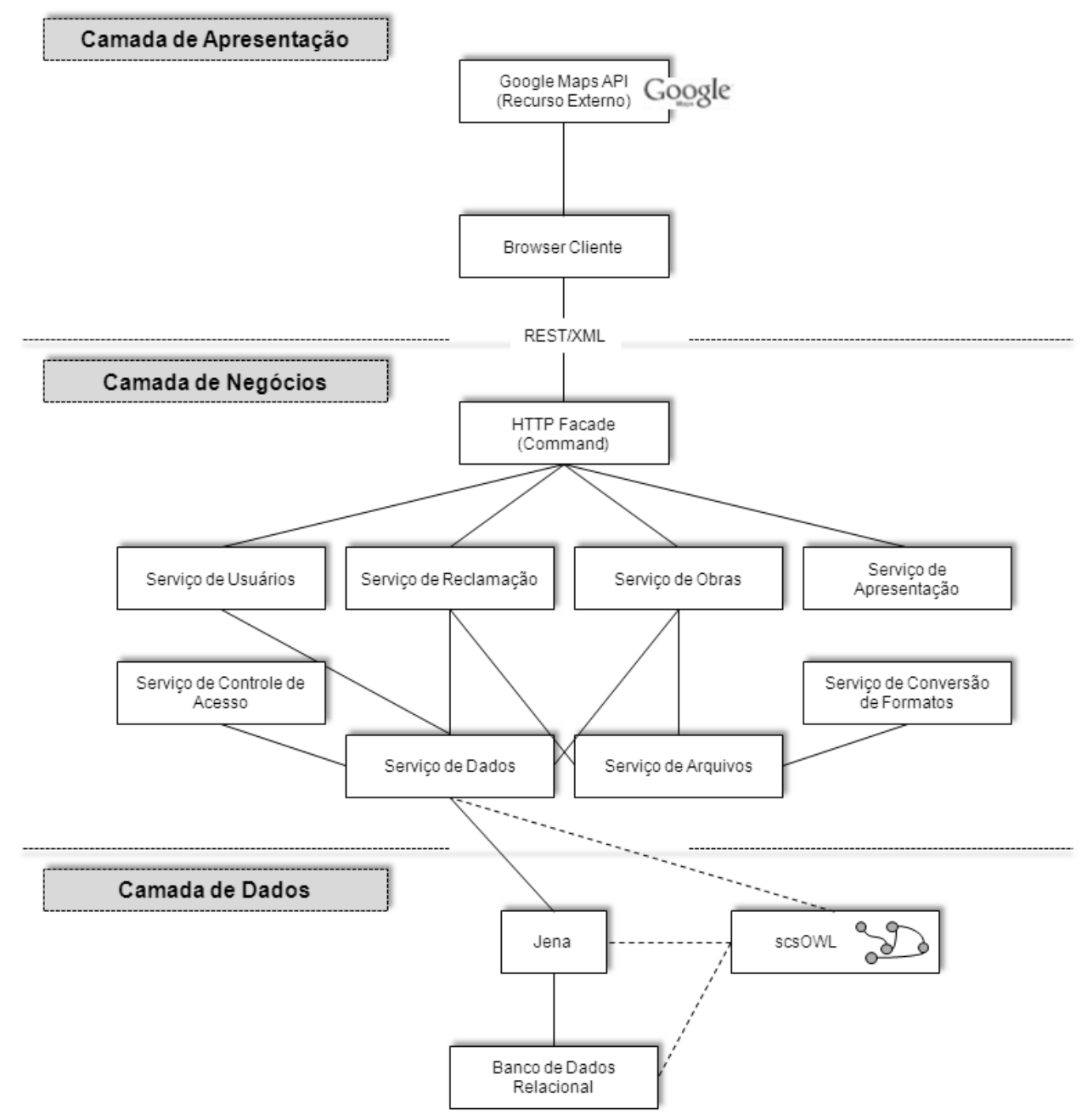

Figura 2. Arquitetura do SocialSupervisor.

A primeira camada é a de Apresentação, nela o usuário tem acesso as interfaces presentes no sistema através dos browsers dos computadores. Esta camada foi construída utilizando a tecnologia Flex [Flex] e também o serviço de mapas do Google, para poder manipular de forma visual o espaço geográfico das obras.

A segunda camada é a de Negócios, onde se encontra toda a lógica de negócios utilizada no sistema, isso significa que todas as solicitações feitas pelo usuário a camada de Apresentação são processadas por esta camada. No SocialSupervisor, os módulos que compõem esta camada são: i) o serviço de apresentação, responsável por processar os resultados que serão apresentados ao usuário; ii) o serviço de denúncia, que gerencia as denúncias realizadas no sistema; iii) o serviço de obras, que possui a função de gerenciar as obras públicas que estão cadastradas no sistema; e iv) o serviço de usuários, responsável por gerenciar os usuários do sistema. Além destes, ainda fazem parte da camada de Negócios os módulos que monitoram as atividades dos usuários (serviço de controlo de acesso) e que formatam o resultado de uma requisição de forma 
adequada (serviço de conversão de formatos). Por fim, existem os serviços de dados e de arquivos, responsáveis respectivamente por prover acesso ao banco de dados e ao sistema de arquivos.

A comunicação entre a camada de Apresentação e a camada de Negócios é implementada através do REST (Representational State Transfer) e do XML.

A terceira e última camada que compõe o modelo de três camadas é a de Dados, responsável pela comunicação entre a camada de Negócios e os mecanismos de armazenamento dos dados. Esta comunicação é realizada utilizando o framework Jena [Jena], que por sua vez tem acesso ao modelo da ontologia.

\subsection{Cadastro e Denúncia de Obras Públicas}

O cadastro de obras públicas no SocialSupervisor é restrito a usuários com autorização, como por exemplo, os fiscais de obra do órgão público. A interface utilizada para registrar obras é apresentada na Figura 3. Nela é possível observar que é necessário cadastrar alguns dados a respeito da obra, além de sua localização geográfica, que pode ser selecionada de forma visual, através de um mapa (não apresentado na Figura) e também é possível anexar arquivos multimídia, como fotos e vídeos que mostrem a obra. A representação da localização geográfica de uma obra pode ser um ponto, ou um conjunto de pontos.

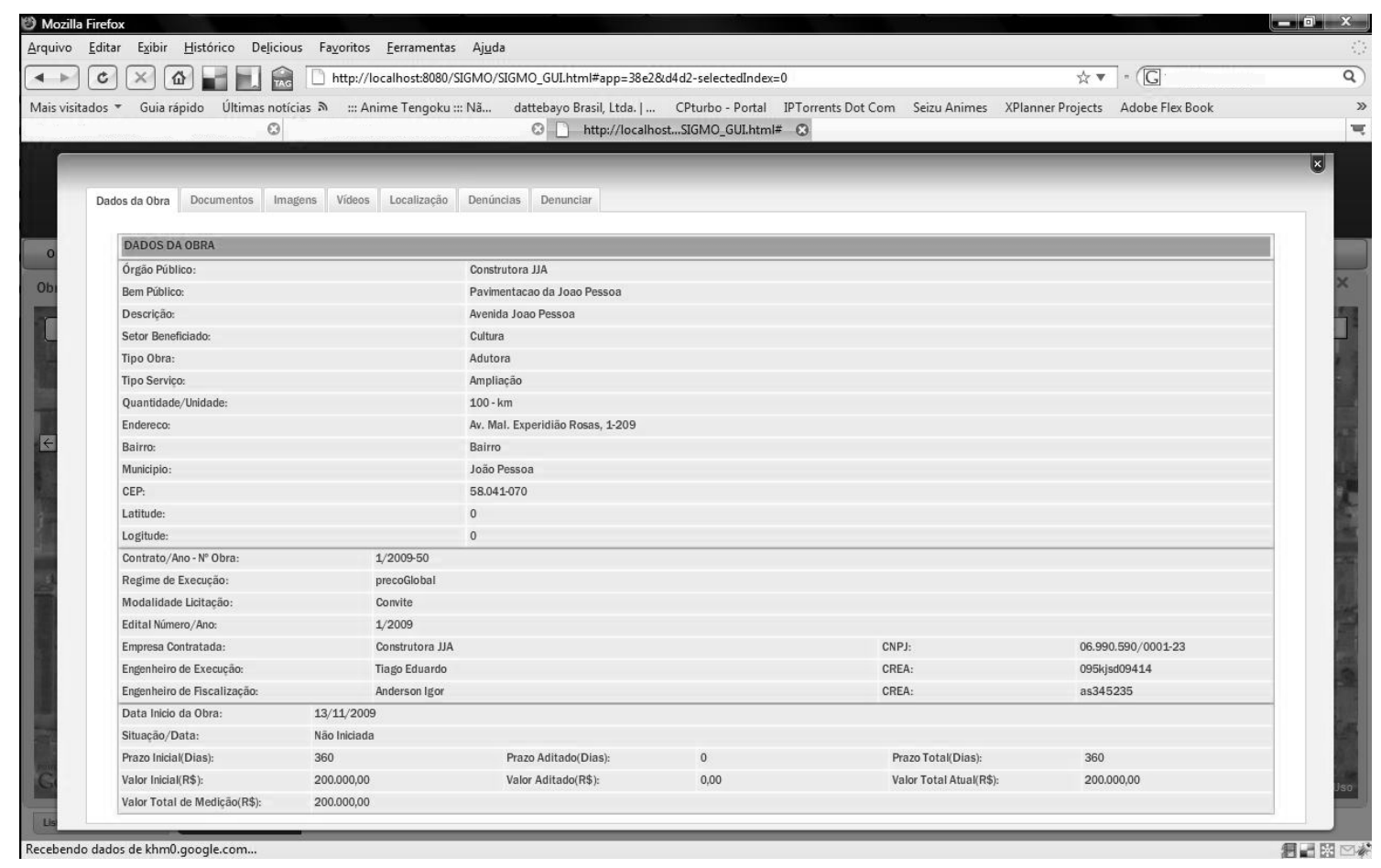

Figura 3. Interface de cadastro de obras públicas no SocialSupervisor. 


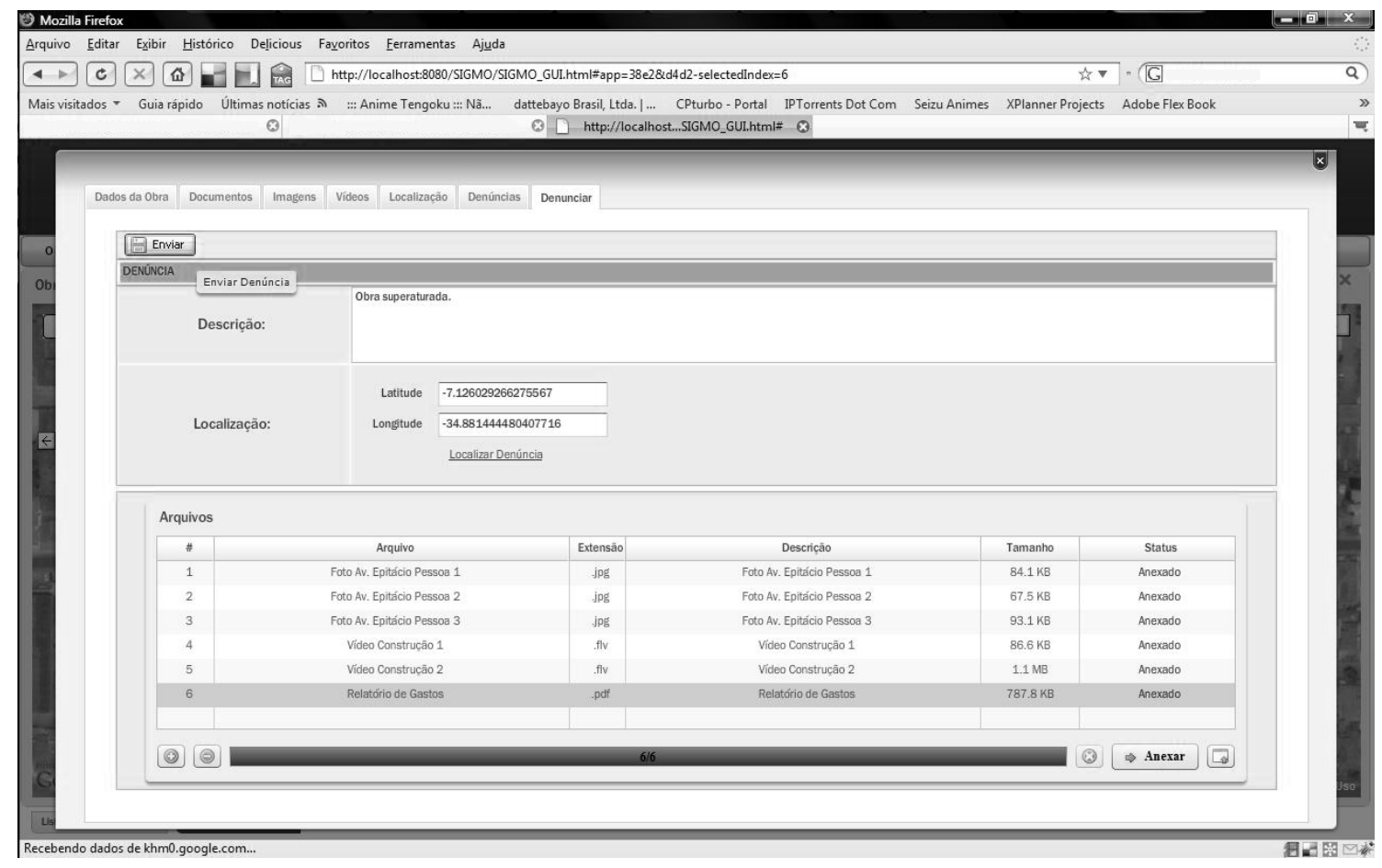

Figura 4. Interface de denúncias de obras públicas do SocialSupervisor.

Para a realização de denúncias a respeito de uma obra cadastrada no sistema o usuário utiliza a interface mostrada na Figura 4. Nela, é possível descrever o motivo da denúncia, anexar arquivos multimídia e informar a localização geográfica onde o problema está ocorrendo.

\section{Veracidade das Denúncias e Fotografias no SocialSupervisor}

Uma questão importante a ser considerada nas denúncias é a sua veracidade, já que no SocialSupervisor é permitido realizá-las anonimamente. A solução utilizada no sistema foi a utilização de moderadores, que são pessoas autorizadas a avaliar as queixas, a fim de aceitá-las ou rejeitá-las. As denúncias aceitas automaticamente passam a ser visualizadas em um mapa juntamente com as obras.

A Figura 5 mostra um mapa com obras públicas e denúncias. Nele, os ícones de número 1 indicam a localização geográfica das denúncias e os numerados com 2 apresentam a posição geográfica das obras cadastradas. Quando mais de um ponto é utilizado para definir a localização espacial de uma obra, o mapa mostra além do ícone, a geometria formada pelos pontos. Na Figura 5, por exemplo, a linha entre as letras A e $\mathrm{B}$, marca os pontos utilizados para representar uma obra do tipo rodovia, e a letra $\mathrm{C}$ mostra o local onde foi realizada uma denúncia a respeito desta obra. 


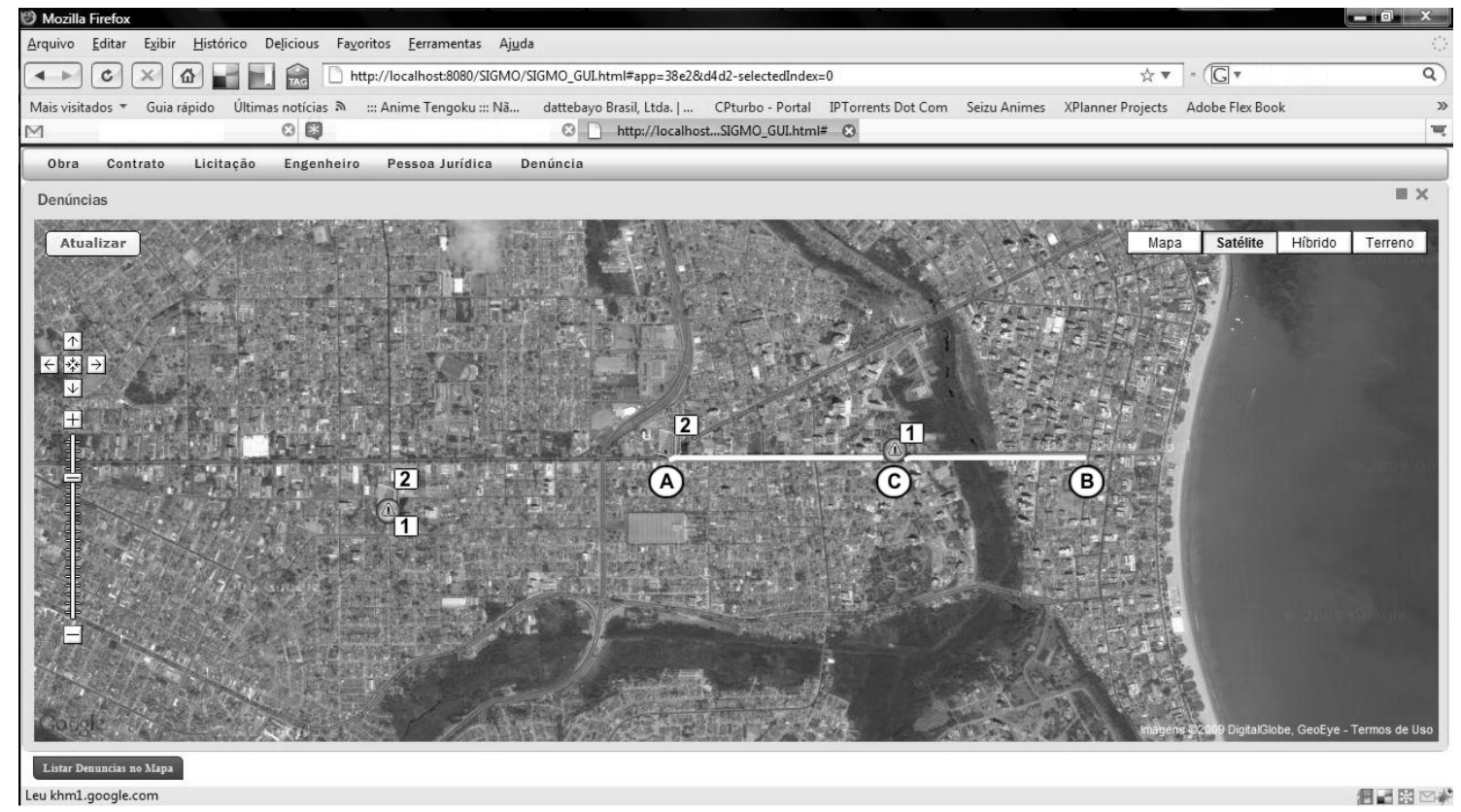

Figura 5. Interface de visualização de obras públicas e denúncias do SocialSupervisor.

Uma das formas de apresentar a sociedade o progresso de uma obra é utilizando fotografias que caracterizem as diversas fases da construção. Essas fotografias são inseridas no SocialSupervisor por usuários autorizados, mas a aquisição delas, muitas vezes não é realizada por pessoas diretamente relacionadas ao órgão fiscalizador. Desta forma podem acontecer casos em que as fotografias publicadas não condizem com a realidade da obra. Para ajudar a identificar a veracidade das fotos que são publicadas no SocialSupervisor um cidadão que mora próximo a determinada obra pode verificar se as fotos publicadas representam de fato o estágio atual da construção.

\section{Motivação para novas aplicações}

A Web 2.0 explora a inteligência coletiva para produzir ferramentas mais aprimoradas para o usuário, como é o caso dos Blogs e Wikis, com os quais se pode facilmente compartilhar experiências e conhecimentos.

$\mathrm{Na}$ fiscalização de obras públicas, a utilização de uma ferramenta que agregue características da Web 2.0 possibilitará a participação mais efetiva da população, uma vez que as pessoas que moram próximas a uma determinada obra podem utilizar a ferramenta para expor reclamações, opiniões ou simplesmente comparar a veracidade das informações publicadas a respeito da construção com o que é visto na prática. Apesar do monitoramento e fiscalização da obra ser de responsabilidade do governo, este pode utilizar-se da ajuda do cidadão para identificar irregularidades existentes. Esta participação da sociedade na fiscalização da aplicação do dinheiro público tem sido chamada de controle social.

No Brasil, existem agências especializadas para receber reclamações a respeito de irregularidades em obras públicas. Entretanto, os canais de comunicação disponibilizados, tais como o telefone, possuem limitações. Com a utilização dos recursos da Web 2.0, essas limitações seriam minimizadas, tornando mais eficiente a 
identificação de possíveis problemas, mantendo também o anonimato do denunciante, caso seja requerido.

Os conteúdos publicados na Web social nem sempre são verdadeiros. Desta forma, para tentar aumentar o nível de confiança da informação, podem ser feitas auditorias, por representantes do órgão que fiscaliza as obras, através de inspeções, pelos usuários da Web, através de votação a respeito de uma denúncia realizada ou de uma maneira hibrida, pelos representantes e usuários.

Atualmente, as vantagens da Web 2.0 também foram incorporadas aos dispositivos móveis, sendo este novo paradigma denominado Mobile 2.0. Os aparelhos móveis mais modernos permitem que o usuário tenha acesso a Internet, capture fotografias, vídeos e áudios e muitos também possuem sensores, como o GPS (Global Positioning System), que é capaz de recuperar informações a respeito da localização geográfica do aparelho.

Aplicando o paradigma Mobile 2.0 aos dispositivos móveis, este se torna uma importante ferramenta de compartilhamento de informações, podendo ser utilizado para enviar denúncias a respeito de obras públicas, publicando fotos, vídeos e até mesmo informações geográficas.

\section{Conclusão e Trabalhos Futuros}

Este artigo apresentou uma proposta de modelo baseado em ontologias para realizar a modelagem semântica e geográfica de sites de conteúdo social no contexto dos setores públicos. Para desenvolver o modelo foram reusadas ontologias e vocabulários bastante conhecidas no âmbito da web semântica, tais como FOAF e MOAT, GeoOWL e SIOC. A combinação destes modelos resultou no scsOWL, ontologia que mapeia as interações dos usuários em um site de conteúdo social e possibilita que sejam utilizadas tags com marcações semânticas e geográficas para descrever os significados e a localização dos recursos.

Também foi mostrado o protótipo construído utilizando o modelo proposto: o SocialSupervisor, um site de conteúdo social enriquecido geograficamente, que tem o objetivo de melhorar a comunicação entre os cidadãos e os órgãos responsáveis por supervisionar obras públicas. No sistema construído, qualquer pessoa pode acessar as informações sobre as obras públicas cadastradas, e realizar denúncias. No protótipo, para melhor explorar as informações presentes no site e aumentar a percepção do usuário a respeito do que está acontecendo em determinada região geográfica, são utilizados recursos da Web 2.0 e mashups de mapas.

Como trabalho futuro, pretende-se criar um protótipo utilizando dispositivos móveis e o modelo proposto de ontologia também no contexto da supervisão de obras públicas. Além disso, a ontologia scsOWL será aperfeiçoada com o objetivo de poder mapear questões a respeito da veracidade dos recursos que são colocados no site de conteúdo social e também uma modelagem para capturar a interação a respeito de votações que sejam realizadas no site.

\section{Agradecimentos}

Os autores agradecem o CNPQ por financiar esta pesquisa, número do processo 477948/2009-0. 


\section{Referências}

Amer-Yahia, S., Huang, J., and Yu, C.: Jelly: A Language for Building CommunityCentric Information Exploration Applications. IEEE International Conference on Data Engineering, 1588-1594 (2009A).

Amer-Yahia, S., Lakshmanan, L., Yu, C.: SocialScope: Enabling Information Discovery on Social Content Sites. Conference on Innovative Data Systems Research, (2009B).

Berners-Lee, T., Hendler, J., Lassila, O.: The Semantic Web - A new form of Web content that is meaningful to computers will unleash a revolution of new possibilities; Scientific American, Vol. 284, No. 5, May, pp. 35-43. (2001).

Boldi, P., Bonchi, F., Castillo, C., Vigna, S.:Voting in Social Networks. Conference on Information and Knowledge Management, (2009).

Flex: http://www.adobe.com/br/products/flex/

FOAF, Friend of a Friend, http://www.foaf-project.org/

GeoOWL, W3C Geospatial Vocabulary, http://www.w3.org/2005/Incubator/geo/XGRgeo-20071023/

GeoRSS Vocabulary, http://www.georss.org

Goodchild, M. F.: Citizens as sensors: Web 2.0 and The Volunteering of geographic information. GeoFocus, 8-10 (2007).

Guarino, N.: Understanding, building and using ontologies. Proceedings of Knowledge Acquisition for Knowledge-Based Systems Workshop. (1996).

Heidinger, C., Buchmann, E., Böhm, K.: Towards collaborative web-based impact assessment. Proceedings of the 10th Annual International Conference on Digital Government Research, 190-198 (2009).

Jena, Semantic Web Framework, http://jena.sourceforge.net

Maciel, C., Roque, L., Garcia, A. C. B.: Democratic citizenship community: a social network to promote e-deliberative process. Proceedings of the 10th Annual International Conference on Digital Government Research, 25-34 (2009).

Merrill, D.: Mashups: The new breed of Web app. IBM Web Architecture Technical Library, http://www-128.ibm.com/developerworks/library/x-mashups.html (2006).

Monclar, R., Tecla, A., Oliveira, J., de Souza, J.M.: MEK: Using spatial-temporal information to improve social networks and knowledge dissemination. Information Sciences, 2524-2537 (2009).

Naaman, M.: Spatio-Tempo-Social: Learning from and about Humans with Social Media. Proceedings of the 11th International Symposium, Advances in Spatial and Temporal Databases, 1-2 (2009).

Newman, R.: Tag ontology design, http://www.holygoat.co.uk/projects/tags/, (2005).

Noveck, B.: Social Networks: Making Connections between Citizens, Data and Government. Proceedings of the 10th Annual International Conference on Digital Government Research, 1-1 (2009). 
Passant, A., Laublet, P.:Meaning Of A Tag: A collaborative approach to bridge the gap between tagging and Linked Data. Proceedings of the WWW 2008 Workshop Linked Data on the Web, Beijing, China (2008).

Rodriguez, M., Steinbock, D., Watkins, J., Gershenson, C., Bollen, J., Grey, V., and deGraf, B.:Smartocracy: Social Networks for Collective Decision Making. Hawaii International Conference on Systems Science, (2007).

Sheth, A., and Nagarajan, M.: Semantics-Empowered Social Computing. IEEE Internet Computing , 76-80 (2009).

SIOC, Semantically Interlinked Online Communities, http://sioc-project.org/.

Teerling, M. L., Pieterson, W.: Multi-channel marketing: an experiment on leading citizens to online public services. Proceedings of the 10th Annual International Conference on Digital Government Research, 104-112 (2009). 